\title{
Aicardi Syndrome in a 8 month Old Female Child
}

\section{Edwin Dias}

Adjunct Professor, College of Allied Health Sciences, Srinivas University, Mangalore, India. Professor and HOD, Department of Pediatrics, Srinivas Institute of Medical Sciences and

Research Centre, Mangalore, India.

Email: edwindias@gmail.com

Type of the Paper: Case Report.

Type of Review: Peer Reviewed.

Indexed In: OpenAIRE.

DOI: https://doi.org/10.5281/zenodo.2646424.

Google Scholar Citation: IJHSP

\section{How to Cite this Paper:}

Dias, Edwin. (2019). Aicardi Syndrome in a 8 month Old Female Child. International Journal of Health Sciences and Pharmacy (IJHSP), 3(1), 18-20.

DOI: https://doi.org/10.5281/zenodo.2646424.

International Journal of Health Sciences and Pharmacy (IJHSP)

A Refereed International Journal of Srinivas University, India.

(C) With Author.

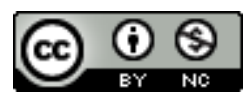

This work is licensed under a Creative Commons Attribution-Non Commercial 4.0 International License subject to proper citation to the publication source of the work.

Disclaimer: The scholarly papers as reviewed and published by the Srinivas Publications (S.P.), India are the views and opinions of their respective authors and are not the views or opinions of the SP. The SP disclaims of any harm or loss caused due to the published content to any party. 


\title{
Aicardi Syndrome in a 8 month Old Female Child
}

\author{
Edwin Dias \\ Adjunct Professor, College of Allied Health Sciences, Srinivas University, Mangalore, India. \\ Professor and HOD, Department of Pediatrics, Srinivas Institute of Medical Sciences and \\ Research Centre, Mangalore, India. \\ Email: edwindias@gmail.com
}

\begin{abstract}
Aicardi syndrome (AS) is a triad of infantile spasms, chorioretinal 'lacunae' and agenesis of corpus callosum. The exact frequency of this condition is not known. Here we provide a description of an 8 month old child with Aircardi syndrome. Treated with multiple Antiepileptic drugs.
\end{abstract}

Keywords: Aicardi syndrome, Infantile spasms, Antiepileptics.

\section{INTRODUCTION :}

Aicardi syndrome (AS) was originally described as featuring a triad of infantile spasms, chorioretinal 'lacunae' and agenesis of the corpus callosum. The condition has been recognized only in individuals with two $\mathrm{X}$ chromosomes. Aicardi syndrome is not a familial condition. The other occasional findings are abnormalities of ribs and spine, microphthalmia, optic nerve coloboma, cortical heterotropia, Retro-orbital cyst, some form of speech abnormalities and language impairment is noted in all the available cases. The actual frequency of the condition is not known. In series of infantile spasms from tertiary referral centers, $1 \%$ to $4 \%$ of cases may be due to Aicardi syndrome. Incidence of Aicardi syndrome is estimated about 1 in every 100,000 live birth. Early cases of the syndrome were diagnosed by air encephalography which explains the diagnostic importance attributed to callosal agenesis that was the major recognizable sign using this technique. Modern neuroimaging, especially magnetic resonance imaging (MRI), has shown that a complex brain malformation is more characteristic than isolated agenesis of the callosum which does not occur in all cases. The use of modern neuroimaging and the accumulation of new cases have also led to the recognition of some new clinical signs and to the realization that the course of the disorder, although usually very severe, was in some cases significantly milder. We present a case of Aicardi syndrome in 8 month old female child with a brief review of this rare syndrome.

\section{CASE REPORT :}

A 8 month old child presented with history of flexion of both upper limbs and lower limbs and neck since the age of 3 months, sudden onset, also transient staring in upward direction during these episodes, around seven to eight times a day. She had not been treated for the condition till seven month. Child did not develop any milestones except head control and social smile. There was no weakness of limbs. Feeding, immunization history and family history were not significant. On examination, Vitals were stable. Weight $8 \mathrm{~kg}$, Head circumference $-43 \mathrm{~cm}$, Length $65 \mathrm{~cm}$. No dysmorphic facies or neurocutaneous markers. Eyes were normal. Other general examination did not reveal any significance. Visual acuity and hearing were normal. CNS examination, cranial nerves were normal, motor system showed normal power as per age, intermittent infantile spasms were present. Child is unable to sit. Cranial spinal axis was normal. Other neurological examination did not reveal anything significant. On ophthalmological evaluation choreoretinal lacuna was found.

Investigations- $\mathrm{CBC}$, serum electrolytes were normal. EEG showed high voltage sharp waves with bilateral bursts independent between left and right hemispheres. CT scan showed agenesis of corpus callosum.

Child was treated with Sodium Valproate and Phenobarbitone. Neurosurgery consultation confirmed the diagnosis. The myoclonus still persisted but few episodes. On follow up child continued to have infantile spasms in spite 
$\begin{array}{llr}\text { multiple } & \text { antiepileptic treatment, } \\ \text { developmental } & \text { regression } & \text { was also }\end{array}$ documented.

\section{DISCUSSION :}

The patient affected by Aicardi syndrome shows a severe neurodegenerative encephalopathy and subsequent motor and mental delay [1]. The presence of classical triad of infantile spasm, dysgenesis of corpus callosum and chorioretinal lacunae is diagnostic of Aicardi syndrome [2]. The presence of two classic features plus atleast two other major or supporting features is highly indicative of diagnosis [3].

The major features include- polymicrogyria or pachygyria, intra-cranial cyst, heterotopias and optic disc, retinal or choroidal coloboma, vertebral and rib anomalies, microphthalmia, split brain EEG are included in the supporting features. The neurological abnormalities include microcephaly, developmental delay, mental retardation, intractable epilepsy, limb hypertonia with spasticity. The skeletal anomalies such as fused vertebra, hemivertebra, blocked vertebra, absent ribs could be present which may lead to scoliosis. The posterior fossa abnormalties include unilateral hypoplasia or dysplasia of the cerebellar hemisphere with associated large cysterna magna, hypoplastic cerebellar vermis, extraaxial cyst and tectal enlargement. Tectum can be measured in mid sagittal plain. Cut off values for tectal enlargement are length more than $15 \mathrm{~mm}$, width more than $5 \mathrm{~mm}$. aicardi syndrome appears to be inherited in an Xlinked dominant pattern, owing to mutant gene on the $\mathrm{X}$-chromosome that is lethal in $\mathrm{XY}$ male [4].

All the cases are sporadic, not promising due to new mutation $[5,6]$. The most affected children have 46xx karyotype. Only two cases are reported in literature in males. One of them have 46XXY and other had 46XX karyotype. The analysis of Aicardi syndrome is based on clinical facial appearances including the pathognomonic chorioretinal lacunae identified on ophthalmic examination, brain MRI finding and skeletal finding [7]. The management of Aicardi syndrome requires anti-epileptic drugs for seizure control; improved outcome with vigabatrin has been reported. Seizures start early in Aicardi syndrome and have always been myoclonic in nature.

\section{CONCLUSION :}

Aicardi syndrome in an 8 month old female child is a diagnostic and management challenge and often requiring continuous antiepileptic treatment. It has a poor prognosis.

\section{REFERENCES :}

[1] Pedley T.A., Meldrum B Chevrie JJ, Aicardi J (1986) The Aicardi syndrome. in Recent advances in epilepsy. Eds Pedley T.A., Meldrum B (Churchill Livingstone, Edinburgh), 3:189-210.

[2] Donnenfeld, A. E., Packer, R. J., Zackai, E. H., Chee, C. M., Sellinger, B., \& Emanuel, B. S. (1989). Clinical, cytogenetic, and pedigree findings in 18 cases of Aicardi syndrome. American Journal of Medical Genetics, 32(4), 461-467.

[3] Guadagni, M. G., Faggella, A., Piana, G., \& D’Alessandro, G. (2010). Aicardi syndrome: a case report. Eur J Paediatr Dent, 11, 146-8.

[4] Le Garrec, M., Doret, M., Pasquier, J. C., Till, M., Lebon, P., Buenerd, A., ... Gaucherand, P. (2005). Prenatal diagnosis of Aicardi-Goutières syndrome. Prenatal Diagnosis, 25(1), 28-30.

[5] Lanzi, G., Fazzi, E., \& D’ARRIGO, S. (2002). Aicardi-Goutières syndrome: a description of 21 new cases and a comparison with the literature. European Journal of Paediatric Neurology, 6, A9A22.

[6] Melbourne-Chambers, R., Singh Minott, I., Mowatt, L., Johnson, P., \& Thame, M. (2007). Aicardi syndrome associated with anterior cephalocele in a female infant. Developmental Medicine and Child Neurology, 49(6), 464-466. https://doi.org/10.1111/j.14698749.2007.00464.x

[7] Iturralde, D., Meyerle, C. B., \& Yannuzzi, L. A. (2006). Aicardi syndrome: chorioretinal lacunae without corpus callosum agenesis. Retina, 26(8), 977-978. 